\title{
Technical feasibility of robot-assisted laparoscopic radical prostatectomy in renal transplant recipients: Results of a series of 12 consecutive cases
}

\author{
Quentin-Côme Le Clerc, MD; Emilie Lecornet, MD; Gregoire Leon, MD; Jerome Rigaud, MD; \\ Pascal Glemain, MD; Julien Branchereau, MD; Georgess Karam, MD
}

Clinique Urologique, CHU Nantes Hôtel-Dieu 1 Place Alexis Ricordeau, Nantes Cedex

Cite as: Can Urol Assoc J 2015;9(7-8): E490-3. http://dx.doi.org/10.5489/cuaj.2319 Published online July 17, 2015.

\section{Abstract}

Introduction: We evaluate the technical feasibility of robotic prostatectomy in renal transplant recipients.

Methods: We retrospectively analyzed preoperative and perioperative settings, as well as functional and oncologic results of 12 patients operated on between 2009 and 2013. Prostatectomy was performed via a transperitoneal approach without any changing in the ports position. The average age was $61.92 \pm 2.98$ years. The period between transplant and the diagnosis of adenocarcinoma was 79.7 months. The mean PSA was 7.34 ng/mL (range: 4.9-11). Results: The operative time was $241.3 \pm 35.6$ minutes with only one conversion and one transfusion. The intervention was difficult due to adhesions on the side of the graft in $50 \%$ of cases. There was a case of obstructive acute renal failure resulting from a hematoma of the Retzius treated by percutaneous nephrostomy at D20. There was a majority of pT2c $(72.7 \%)$, including 3 positive margins $(27.3 \%)$ and 2 biochemical relapses treated with radiotherapy and hormonotherapy, respectively. The end point prostate-specific antigen was undetectable. There was no significant difference between preoperative and $\mathrm{J} 7$ creatinine $(p=0.22)$.

Conclusions: Robotic prostatectomy in renal transplant recipients is a safe technique with no serious effects on the allograft.

\section{Introduction}

For patients with end-stage renal disease, renal transplantation is the best treatment in terms of patient survival and quality of life. ${ }^{1}$ However, the need for immunosuppressive therapy following transplantation increases the incidence of certain cancers, ${ }^{2}$ including urological cancers. ${ }^{3}$ Prostate cancer is the most common urological cancer in male renal transplant recipients, with an incidence of $3.1 \%{ }^{3}$

The functional and oncological results of radical prostatectomy in these patients remain poorly evaluated. The results of retropubic ${ }^{4,5}$ and laparoscopic prostatectomy have been previously reported, ${ }^{6-8}$ but few studies have evaluated robot-assisted laparoscopic prostatectomy. ${ }^{9}$ We therefore evaluated the technical feasibility and oncological results of robot-assisted laparoscopic radical prostatectomy (RALP) in renal transplant recipients.

\section{Methods}

This single-centre retrospective study included 12 renal transplant recipients who underwent RALP between January 2009 and January 2013.

Clinicom 2001 version 6.50.0102 (Cliniom, Inc.) was used for data collection and Fusion Pégase 4D version 4.0.0 (Fusion Pégase Inc.) was used for anesthetic data. RALP was performed according to the conventional technique with 6 transperitoneal trocars. Lymph node dissection was not performed on the side of the transplant.

Clinical data (age at the time of prostatectomy, etiology of end-stage renal disease, interval between renal transplantation and prostatectomy, side of transplantation, immunosuppressive therapy, American Society of Anesthesiologists [ASA] score, body mass index [BMI], and Charlson comorbidity index) were recorded for each patient.

The diagnosis of prostate cancer was established on the basis of digital rectal examination (DRE), prostatespecific antigen (PSA) assay, and ultrasound-guided prostate biopsy according to the protocol of 12 random biopsies after fluoroquinolone and metronidazole antibiotic prophylaxis. Biopsies were realized in all cases under local anesthesia. Staging comprised bone scan and pelvic magnetic resonance imaging (MRI). Preservation of neurovascular bundles was decided preoperatively case by case on the basis of clinical data and the presence or absence of erections.

Intraoperative events, operating time, blood loss and any operating difficulties, whether or not they were attributed to the presence of the renal transplant, were recorded. 
Infectious and bleeding complications, serum creatinine on day 1 and during follow-up, modification of immunosuppressive therapy, long-term PSA and adjuvant therapies were recorded. Statistical analysis was performed with BiostaTGV software using a Wilcoxon's test for paired data.

\section{Results}

The mean patient age at the time of prostatectomy was $61.92 \pm 2.98$ years (range: $55-73$ ) (Table 1). Most cases of end-stage renal disease were secondary to familial polycystic kidney and liver disease (41.7\%). The mean interval between renal transplantation and RALP was 79.7 months (range: 17-242). The transplant was situated on the left side in most patients (8/12). Only 1 patient was treated by hemodialysis for graft dysfunction (transplanted in 1989).

Immunosuppressive therapy (Table 2) consisted of a combination of calcineurin inhibitor $(91.7 \%)$, antimetabolite $(83.3 \%)$, and corticosteroids (41.7\%). The mean American Society of Anesthesiologists (ASA) score was 2.7 (range: 2-3). The mean BMI was $26.75 \mathrm{~kg} / \mathrm{m}^{2}$ (range: $21-32$ ). The mean PSA was $7.34 \mathrm{ng} / \mathrm{mL}$, with a normal DRE for 7 out of 12 patients (Table 3).

Biopsies demonstrated a Gleason score of $6(3+3)$ for 8 of the 12 cases and a Gleason score of 7 for 4 cases. Bone scan was performed for 8 patients. Prostate MRI, performed in only 6 patients, showed hypointense anomalies with signs of possible extracapsular extension in one case (Table 3).

The mean operating time was $241.3 \pm 35.6$ minutes (range: 178-321), with a mean volume of blood loss of $587.9 \pm 261.3 \mathrm{~mL}$ (only 1 transfused patient). Prostatectomy could not be performed in 1 case, despite conversion to open surgery (patient with a BMI of $32 \mathrm{~kg} / \mathrm{m}^{2}$ presenting major exposure difficulties) (Table 4). This patient was subsequently treated by external beam radiotherapy with no negative impact on renal function or prognosis. The major difficulty observed in this series concerned prostate dissec-

\begin{tabular}{lc}
\hline Table 1. Preoperative clinical characteristics of the patients \\
\hline & $\mathbf{N}=\mathbf{1 2}$ \\
Age at the time of the operation, years & $61.9 \pm 2.98(55-73)$ \\
Time since transplantation, months & 79.7 months (17-242) \\
Etiology of end-stage kidney disease & \\
Polycystic kidney disease & $5(41.7 \%)$ \\
IgA nephropathy & $4(33.3 \%)$ \\
Other & $3(25.0 \%)$ \\
Transplant position & \\
Left iliac fossa & $8(66.7 \%)$ \\
Right iliac fossa & $4(33.3 \%)^{*}$ \\
ASA & $2.7(2-3)$ \\
Charlson comorbidity index & $7.7(5-12)$ \\
BMI (kg/m ${ }^{2}$ ) & $26.75(21-32)$ \\
\hline *Including 1 patient on hemodialysis. ASA: American Society of Anesthesiologists; BMl: \\
body mass index.
\end{tabular}

tion on the side of the transplant due to the presence of numerous adhesions. The vesicourethral anastomosis was difficult in 2 patients due to difficult mobilization of the bladder. No difficulties related to the transplant or the ureterovesical anastomosis were encountered.

Retropubic hematoma was observed on day 10 in 1 patient, requiring drainage and bladder clot removal on day 14 (Table 5). This patient subsequently presented with acute transient renal failure secondary to the hematoma, requiring nephrostomy tube placement. Moreover he experienced transient postoperative brachial plexus palsy after an estimated operating time of 321 minutes.

No significant difference was observed between preoperative serum creatinine (mean: $179.3 \mu \mathrm{mol} / \mathrm{L}$; range: 92-306) and postoperative (day 7$)$ serum creatinine (mean: $170.1 \mu \mathrm{mol} / \mathrm{L}$; range: $93-291)(p=0.22)(T a b l e ~ 6 a$, Table 6b).

Most tumours were stage Pt2c (72.7\%). Positive surgical margins were observed in 4 cases $(36.4 \%$ ) (Table 7 ). Biochemical recurrence was observed in 2 patients. One patient was treated with hormonal therapy and the other treated by external beam radiotherapy to the prostatectomy site. Serum PSA remained undetectable for all patients, including patients with biochemical recurrence with a mean follow-up of 31.2 months (range: 6-55).

\section{Discussion}

Despite the small number of patients in our series, we have found that RALP is safe and effective in renal transplant recipients. The high number of Gleason 6 score is explained by our concern, in the past, not to take an unnecessary risk of cancer progression under immunosuppresion. Since, active surveillance or brachytherapy are proposed when indicated.

The high positive surgical margin rate can be explained by the inclusion of cases with very narrow surgical margins $(50 \%)$. The first RALP was performed by Jhaveri and colleagues in 2008 on a 54-year-old man who had undergone right iliac fossa renal transplantation in 1981. Jhaveri modified the position of the trocars to the position of the transplant and reported the need for extensive disruption of adhesions to allow retropubic dissection. Surgical margins

\begin{tabular}{lcc}
\hline \multicolumn{3}{l}{ Table 2. Immunosuppressive therapy before and after RALP } \\
\hline $\mathbf{N}=\mathbf{1 2}$ & Before RALP & After RALP \\
\hline $\begin{array}{l}\text { A: Calcineurin inhibitors } \\
\text { (Prograf, Advagraf) }\end{array}$ & $11 / 12 *(91.7 \%)$ & $7 / 12(58.3 \%)$ \\
B: Corticosteroids & $5 / 12(41.7 \%)$ & $4 / 12(33.3 \%)$ \\
C: Antimetabolite (Cellcept) & $10 / 12(83.3 \%)$ & $6 / 12(50 \%)$ \\
$\begin{array}{l}\text { D: mTor inhibitors (Rapamune, } \\
\text { Certican) }\end{array}$ & $0 / 12(0 \%)$ & $8 / 12 *(66.7 \%)$ \\
\hline
\end{tabular}

RALP: robot-assisted laparoscopic radical prostatectomy; mTOR: mammalian target of rapamycin. 


\begin{tabular}{lc}
\hline \multicolumn{2}{l}{ Table 3. Diagnostic workup of prostate cancer } \\
\hline Parameters & $\mathbf{N}=\mathbf{1 2}$ \\
\hline DRE & $7(58.3 \%)$ \\
$\quad$ Normal & $2(16.7 \%)$ \\
Adenomatous & $2(16.7 \%)$ \\
Induration & $1(8.3 \%)$ \\
Not specified & $7.34(4.9-11)$ \\
PSA, ng/mL & \\
Biopsies & $8(66.7 \%)$ \\
Gleason 6 (3+3) & $4(33.3 \%)$ \\
Gleason 7 (3+4) & \\
Bone scan & $9(75.0 \%)$ \\
$\quad$ Normal & $1(8.3 \%)$ \\
Not performed & $2(16.7 \%)$ \\
Not specified & \\
Complementary imaging & \\
MRI & $7(58.3 \%)^{*}$ \\
Abnormal & \\
CT (contraindication) & $2(16.7 \%)$ \\
Normal & $3(25.0 \%)$ \\
Not performed & \\
\hline *With 1 case of extracapsular extension. DRE: digital rectal examination; PSA: prostate- \\
specific antigen; MRI: magnetic resonance imaging; CT: computed tomography.
\end{tabular}

were negative with long-term postoperative continence and maintenance of erections. ${ }^{9}$

We did not modify the position of trocars, but surgical margins were higher in our series. This could be related to a lack of experience in RALP at the beginning.

The first laparoscopic radical prostatectomy (LRP) in a 50 -year-old renal transplant recipient was reported by Shah and colleagues. The operation was performed with 4 transperitoneal trocars with an operating time of 215 minutes and an estimated blood loss of $200 \mathrm{~mL}$. Long-term PSA was undetectable. ${ }^{6}$

The Cleveland team retrospectively reviewed 3 cases of LRP in renal transplant recipients with no particular difficulties and with long-term preservation of renal function for 2 patients ( 1 patient required hemodialysis preoperatively). ${ }^{?}$

Maestro and colleagues in 2009 described the transperitoneal operative technique and presented a review of the literature based on 8 renal transplant recipients operated by LRP with no cases of positive surgical margins or modifications of serum creatinine. ${ }^{8}$

Robert and colleagues compared LRP in 9 renal transplant recipients and 164 control patients. No significant difference in terms of preoperative characteristics was observed, but the authors nevertheless modified the position of two lateral trocars according to the position of the transplant. ${ }^{10}$ The main complications in this series were two rectal injuries and a longer bladder catheterization time (18 \pm 15.4 days). No significant difference in terms of oncological results was observed (only 1 case of positive surgical margins without biochemical recurrence). At 6 months, only 1 patient required dialysis due to iliac vein thrombosis with exten-

\begin{tabular}{lc}
\hline Table 4. Preoperative parameters & $\mathbf{N}=\mathbf{1 2}$ \\
\hline Operating time, minutes & $253.72 \pm 41.62$ \\
Blood loss, $\mathrm{mL}$ & 646.87 (trace -1200$)$ \\
Transfusion & $1 / 12(8.3 \%)$ \\
& \\
Preservation of neurovascular pedicles & \\
None & $7 / 11$ \\
Unilateral & $2 / 11$ \\
Bilateral & $2 / 11$ \\
Operative difficulties & \\
Adhesions & \\
On side of transplant & $6 / 12(50.0 \%)$ \\
Seminal vesicles & $5 / 12(41.7 \%)$ \\
Vesicourethral anastomosis & $2 / 12(16.7 \%)$ \\
Bladder injury & $1 / 12(8.3 \%)$ \\
Indigo carmine for suspected ureteral & $2 / 12(16.7 \%)$ \\
injury & $1 / 12$ (failure of \\
Conversion & conversion) \\
\hline
\end{tabular}

sion into the transplant vein with no history of lymph node dissection. ${ }^{10}$

Retropubic radical prostatectomy (RRP), according to the technique described by Walsh, has been studied in renal transplant recipients and has been the subject of several publications, including the multicentre Renal Transplantation Committee of French Urological Association study conducted between 1996 and 2007, which compared retropubic prostatectomy between renal transplant recipients $(n=20)$ and a control group $(n=40) .{ }^{4}$ The main significant difference was an earlier age at diagnosis in the renal transplant population (60.4 vs. 64.4 years). No significant difference was observed between the two groups in terms of histology. Intraoperative ureteral section requiring surgical repair by suture was observed in 2 patients. In contrast, renal transplant recipients presented a higher rate of postoperative generalized sepsis $(15 \%$ vs. $2.5 \%)$, suggesting a probable

\begin{tabular}{|c|c|}
\hline & $N=12$ \\
\hline \multicolumn{2}{|l|}{ Postoperative complications } \\
\hline Retropubic hematoma & $1(8.3 \%)$ \\
\hline \multicolumn{2}{|c|}{ Ureteral obstructive nephropathy } \\
\hline Early & $1(8.3 \%)$ \\
\hline Late (3 months) & $1(8.3 \%)^{*}$ \\
\hline Brachial plexus & $1(8.3 \%)$ \\
\hline Trocar incisional hernia & $1(8.3 \%)$ \\
\hline \multicolumn{2}{|c|}{ Course of PSA after RALP $(n=11)$} \\
\hline Undetectable & $9(81.8 \%)$ \\
\hline Recurrence & $2(18.2 \%)$ \\
\hline \multicolumn{2}{|l|}{ Adjuvant therapy $(n=11)$} \\
\hline External beam radiotherapy & $2(18.2 \%)$ \\
\hline Hormone therapy & $1(9.1 \%)$ \\
\hline
\end{tabular}




\begin{tabular}{|c|c|}
\hline \multicolumn{2}{|c|}{$\begin{array}{l}\text { Table 6a. Preoperative-postoperative variation of serum } \\
\text { creatinine }\end{array}$} \\
\hline & Serum creatinine $(\mu \mathrm{mol} / \mathrm{L})$ \\
\hline & $N=12$ \\
\hline Preoperative & $174.2(92-306)$ \\
\hline Day 1 & $169.9(105-282)$ \\
\hline Day 7 & $169.5(93-291)$ \\
\hline \multicolumn{2}{|l|}{ No. patients: $10^{*}$} \\
\hline 6 months & $177.4(91-422)$ \\
\hline 12 months & $223.7(98-665)$ \\
\hline Last serum creatinine & $261.4(123-820)$ \\
\hline
\end{tabular}

effect of immunosuppression. The authors did not report any long-term variation of serum creatinine. Histological examination revealed the presence of positive surgical margins in 2 cases with 2 cases of long-term biochemical recurrence. Thirteen patients achieved total continence (65\%).

Hoda and colleagues in a series of 16 patients reported the feasibility of RRP in renal transplant recipients versus a control group, with a significant difference in terms of operating time (108 vs. 89.1 minutes) and intraoperative blood loss (349 vs. $211 \mathrm{~mL}$ ). One case in their series presented positive surgical margins without biochemical recurrence. Long-term serum creatinine remained stable. ${ }^{5}$

In other series of RRP, 10 of 17 patients had Gleason 6 score $(62.5 \%)$ and only 1 biochemical recurrence reported $(5.88 \%) .{ }^{11}$

However, the 3D conformal technique has been reported by Mouzin and colleagues, with 2 patients showing isolated biochemical recurrence ( $25 \%$ ), with a mean follow-up of 28 months. $^{12}$

\section{Conclusion}

RALP is feasible in renal transplant recipients, with no major modification of the surgical technique and no negative impact on renal function and prognosis.

\begin{tabular}{lc}
\hline Table 7. Histological results & \\
\hline No. patients: $\mathbf{1 1}$ & $\mathbf{N}=\mathbf{1 2}$ \\
\hline Weight of prostate (grams) & $45.96(21-64)$ \\
Adenocarcinoma & \\
Well differentiated & $4(36.4 \%)$ \\
Moderately differentiated & $4(36.4 \%)$ \\
Poorly differentiated & $3(27.3 \%)$ \\
Gleason score & \\
Gleason $6(3+3)$ & $5(45.5 \%)$ \\
Gleason 7 (3+4) & $6(54.5 \%)$ \\
Positive surgical margins & $4(36.4 \%)$ \\
Stage & \\
PT2a & $0(0 \%)$ \\
PT2b & $1(9.1 \%)$ \\
PT2c & $8(72.7 \%)$ \\
PT3a & $2(18.2 \%)$ \\
\hline
\end{tabular}

\begin{tabular}{lc}
$\begin{array}{l}\text { Table 6b. Preoperative-postoperative variation of creatinine } \\
\text { clearance }\end{array}$ \\
\hline \multicolumn{2}{l}{ Creatinine clearance MDRD (mL/min) } \\
\hline & $\mathrm{N}=12$ \\
Preoperative & $45.9(18.8-69.9)$ \\
Day 1 & $45.3(20.6-69.2)$ \\
Day 7 & $47.5(19.9-77.2)$ \\
No. patients: $10^{*}$ & \\
6 months & $46(12.9-73.4)$ \\
12 months & $40(7.7-65.6)$ \\
Last serum creatinine & $40.2(6-68.1)$ \\
\hline${ }^{*}$ Missing data for 1 patient.
\end{tabular}

Competing interests: The authors declare no competing financial or personal interests.

This paper has been peer-reviewed.

\section{References}

1. Schnuelle P, Lorenz D, Trede $M$, et al. Impact of renal cadaveric transplantation on survival in end-stage renal failure: evidence for reduced mortality risk compared with hemodialysis during long-term follow-up. J Am Soc Nephrol 1998;9:2135-41.

2. Penn I. Occurrence of cancers in immunosuppressed organ transplant recipients. Transplant Proc 2002;34:722. http://dx.doi.org/10.1016/S0041-1345(02)02624-6

3. U.S. Renal Data System. Atlas of End-Stage Renal Disease in the United States, National Institutes of Health, National Institute of Diabetes and Digestive and Kidney Diseases. USDRDS 2003 Annual Data Report 2003.

4. Kleinclauss FM, Neuzillet $Y$, Tillou $X$, et al. Morbidity of retropubic radical prostatectomy for prostate cancer in renal transplant recipient: Multicenter study from Renal Transplantation Committee of French Urological Association. Urology 2008;72:1366-70. http://dx.doi.org/10.1016/j.urology.2008.03.018

5. Hoda MR, Hamza A, Greco F, et al. Management of localized prostate cancer by retropubic radical prostatectomy in patients after renal transplantation. Nephrol Dial Transplant 2010;25:3416-20. http:// dx.doi.org/10.1093/ndt/gfq193

6. Shah KK, Ko DS, Mercer J, et al. Laparoscopic radical prostatectomy in a renal allograft recipient. Urology 2006;68:672.e5-e7. http://dx.doi.org/10.1016/i.urology.2006.03.029

7. Thomas AA, Nguyen NM, Gill IS. Laparoscopic transperitoneal radical prostatectomy in renal transplant recipients: A review of three cases. Urology 2008;71:205-8. http://dx.doi.org/10.1016/j.urology.2007.10.017

8. Maestro MA, Gomez AT, Alonso YGS, et al. Laparoscopic transperitoneal radical prostatectomy in renal transplant recipients: A review of the literature. BJU Int 2010;105:844. http://dx.doi.org/10.1111/ j.1464-410X.2009.08911.x

9. Jhaveri JK, Tan GY, Scherr DS, et al. Robot-assisted laparoscopic radical prostatectomy in the renal allograft transplant recipient. J Endourol 2008;22:2475-9. http://dx.doi.org/10.1089/end.2008.0280

10. Robert $G$, Elkentaoui $H$, Pasticier $G$, et al. Laparoscopic radical prostatectomy in renal transplant recipients. Urology 2009;74:683-7. http://dx.doi.org/10.1016/i.urology.2009.04.053

11. Thompson RH, Leibovich BC, Karnes RJ, et al. Radical retropubic prostatectomy in immunosuppressed transplant recipients. J Urol 2008;179:1349-52. http://dx.doi.org/10.1016/i.juro.2007.11.054

12. Mouzin $M$, Bachaud JM, Kamar N, et al. Three dimensional conformal radiotherapy for localized prostate cancer in kidney transplant recipients. Transplantation 2004;78:1496-500.

Correspondence: Dr. Georges Karam, Clinique Urologique, CHU Nantes Hôtel-Dieu 1 Place Alexis Ricordeau 44093 Nantes Cedex France; georges.karam@chu-nantes.fr 